\title{
Surface structure of quark stars with magnetic fields
}

\author{
Prashanth Jaikumar* \\ Physics Division, Argonne National Laboratory, Argonne IL 60439, USA.
}

\begin{abstract}
We investigate the impact of magnetic fields on the electron distribution in the electrosphere of quark stars. For moderately strong magnetic fields $B \sim 10^{13} \mathrm{G}$, quantization effects are generally weak due to the large number density of electrons at surface, but can nevertheless affect the spectral features of quark stars. We outline the main observational characteristics of quark stars as determined by their surface emission, and briefly discuss their formation in explosive events termed Quark-Novae, which may be connected to the $r$-process.
\end{abstract}

PACS: 26.60.+c, 24.85.+p, 97.60.Jd

\section{INTRODUCTION}

There is renewed interest in the theory and observation of strange quark stars, which are believed to contain, or be entirely composed of, deconfined quark matter [1]. An observational confirmation of their existence would be conclusive evidence of quark deconfinement at large baryon densities, an expected feature of Quantum Chromodynamics (QCD). Furthermore, discovery of a stable bare quark star affirms the Bodmer-Terazawa-Witten conjecture [2], viz., that at high enough density, strange quark matter, composed of up, down and strange quarks, is absolutely stable with respect to nuclear matter. This intriguing hypothesis is over three decades old, and bare quark stars are but one possible realization put forward in the intervening years. Broadly speaking, the field of quark star research follows two intertwined paths: (1) efforts to describe the global (mass and radius) and surface structure of such stars from microscopics and (2) determination of observational features that distinguish them from their close cousins, neutron stars. It is possible that a few of the approximately 1500 neutron stars detected thus far are really quark stars [3].

A positive identification of a quark star would likely require a complement of consistent signals. For example, physical properties such as maximum mass, radius, minimum spin-periods and neutrino/photon cooling rates (and hence temperature) all depend on the (unique) equation of state for dense quark matter. As the latter is uncertain, there is a range of allowed values for the above quantities that makes a distinction based on current observational data difficult. We emphasize that a bare strange star is selfbound $[2,4]$ while a neutron star requires gravitational forces for its binding. This implies a very different equation of state for the two classes of stars; however, in the range of masses $\left(1.35<M / M_{\odot}<2.4\right)$ observed to date, the calculated radii are similar $(R \sim 10$ to $15 \mathrm{~km})$. Perturbative corrections to the quark matter equation of state and color superconductivity effects further complicate the issue [5]. ${ }^{1}$

It was first pointed out by Alcock et al. [11] that the bare quark star surface would be characterized by an abrupt termination of the quark density (density gradient $\sim 10^{26} \mathrm{~g} / \mathrm{cm}^{4}$ ) and a less rapid falloff of a charge neutralizing skin of electrons termed the electrosphere (density gradient $\sim 10^{18} \mathrm{~g} / \mathrm{cm}^{4}$ ). The latter extends up to 1000 Fermis above the bare quark star surface. The electric field which binds these electrons to the surface is large enough to pull electron-positron pairs out of the QED vacuum, prompting copious pair-emission which is directed outward by the radiation output by the star [12]. This mechanism of emission from the bare surface of a strange quark star, due to both photons and $e^{+} e^{-}$ pairs, can produce luminosities well above the Eddington limit $\left(\sim 10^{38} \mathrm{erg} \mathrm{sec}^{-1}\right)$ up to a decade after the quark star's birth [13]. The spectrum of emitted photons is significantly harder than that from a normal cooling neutron star $(30<E / \mathrm{keV}<500$ instead of $0.1<E / \mathrm{keV}<2.5)$. After the star has

\footnotetext{
*jaikumar@phy.anl.gov

${ }^{1}$ We will not elaborate here on the possibility of having superconducting phases of quarks. We refer the interested reader to Refs. [6-10] for a consideration of these phases, and the consequences of their existence inside compact stars in the present context.
} 
cooled to temperatures less than $T<6 \times 10^{8} \mathrm{~K}$, other microscopic processes of photon emission can dominate the luminosity of the star and yield a non-thermal spectrum [14]. This distinctive spectrum and temperature evolution, if observed, would constitute an unmistakable signature of a strange quark star, but would require a fortuitous detection before the star cools below the observational threshold of present-generation space-based laboratories such as INTEGRAL [15].

Quark stars may be much more elusive if they have a suspended crust of normal matter up to the neutron drip density, that is separated from the quark surface by a microscopic gap but supported by the large electric fields at surface [11,16]. Alternatively, quark stars may admit a mixed phase crust several meters in thickness and extending up to its surface, if surface and Coulomb costs (determined at the microscopic level by the current quark mass of the strange quark) is small enough $[17,18]$. This would obviate the need for large electric fields at surface, smoothen the density gradient at surface, and render the previously proffered signals inapplicable. These uncertainties are not expected to be resolved quickly since they involve quantifying the parameters of QCD to better than $5 \%$ in a distinctly nonperturbative regime. (NB. The density that characterizes quark matter in these objects corresponds to a quark chemical potential $\mu_{q} \sim 500 \mathrm{MeV}$ which does not greatly exceed $\Lambda_{\mathrm{QCD}} \sim 200 \mathrm{MeV}$.)

However, we can certainly aim to quantitatively improve our current understanding of the surface structure under the working assumption that the star has a bare quark surface with an electrosphere, since electromagnetic effects dominate over QCD effects. A clear and necessary improvement is the introduction of surface magnetic fields. Neutron stars are born with intense magnetic fields of order $10^{12}$ Gauss or higher, which may decay by over 5 orders of magnitude in the star's lifetime. If a neutron star undergoes a phase conversion to a bare quark star, the surface magnetic fields are expected to remain intact in geometry, unless interior magnetic fields are expelled by a superconducting state that displays the Meissner effect, in which case rapid magnetic reconnections occur, diminishing the field at surface to almost zero [19]. In this work, we will superimpose a static magnetic field $B$ on the electrosphere of a quark star, and calculate the profile for the electrosphere as a first step toward characterizing the spectrum of a magnetized quark star. We expect considerable differences from the $B=0$ case since magnetized atmospheres of neutron stars are already known to be very different from non-magnetized ones [20]. Further, the inclusion of magnetic fields is a step toward a more realistic treatment of the quark star spectrum.

In the next section, we reintroduce the established picture of the electrosphere for the purpose of a self-contained presentation, and mention the modifications due to the strong magnetic field. In section III, we quantify the electrostatic potential and electron number density in the magnetized electrosphere, and state qualitative impacts on the electrospheric photon emission. We conclude in section IV with a general discussion on the formation of Quark stars, with particular attention to the attractive Quark-Nova scenario which may be connected to $r$-process nucleosynthesis.

\section{DEGENERATE ELECTRON GAS IN A STRONG MAGNETIC FIELD}

Recently, a few authors [21] pointed out that the deficit of (massive) strange quarks due to surface effects on the star can lead to a thin charged skin a few Fermis thick, which can lead to a jump in the electric field at surface. The local charge neutrality condition is $2 / 3 n_{u}-1 / 3 n_{d}-1 / 3 n_{s}-n_{e}=0$, with the appropriate low temperature $\left(T \ll \mu_{i}\right)$ expressions in the bulk:

$$
n_{i}=\frac{p_{F, i}^{3}}{\pi^{2}}=\frac{\left(\mu_{i}^{2}-m_{i}^{2}\right)^{3 / 2}}{\pi^{2}} ; \quad m_{e} \ll m_{u, d} \ll m_{s}<\mu_{q} \forall q .
$$

Imposing the conditions of $\beta$-equilibrium and baryon number conservation shows that $\mu_{e} \simeq m_{s}^{2} / 4 \mu$ to better than 1\%. At surface, $s$-quarks are depleted due to surface tension effects by an amount [22]

$$
\delta n_{s}=-\frac{3}{4 \pi} p_{F, s}^{2} \psi\left(\lambda_{s}\right) ; \psi\left(\lambda_{s}\right)=\left[\frac{1}{2}+\frac{\lambda_{s}}{\pi}-\frac{\left(1+\lambda_{s}^{2}\right)}{\pi} \arctan \left(\lambda_{\mathrm{s}}^{-1}\right)\right] ; \lambda_{s}=\frac{m_{s}}{p_{F, s}}
$$

leading to a charged layer at the surface of a bare quark star characterized by a surface charge density $\sigma=-(1 / 3) e \delta n_{s}$ and a discontinuous normal electric field with $\Delta E=4 \pi \sigma$. For "cold" electrospheres, corresponding to the temperature bound $T<5 \times 10^{11} \mathrm{~K}$, the electric field is directed inward just underneath the surface and outward just above it. The electrostatic potential and the electron number density 
are substantially increased cf. the case when surface effects are ignored (NB. The corresponding surface depletion of light quarks is negligible.) [21].

The large electric field leads to the Schwinger instability of the QED vacuum [23], resulting in electronpositron pair-emission, which can be an additional source of photons in the electrosphere, which normally radiates photons via $2 \rightarrow 3$ QED processes. A model cooling calculation of bare strange stars with electrospheric effects and the inclusion of color superconductivity (which can alter the specific heat and thermal conductivity of quark matter) has also been performed [13]. The conclusion is that bare strange stars will display Super-Eddington photon luminosities at surface temperatures $T>10^{9} \mathrm{~K}$, with a spectrum that is hard enough to distinguish it from thermally radiating neutron stars. At lower temperatures $6 \times 10^{8} \mathrm{~K}<T<10^{9} \mathrm{~K}$, the bulk of the luminosity comes from electron-positron pairs which subsequently imprint a wide annihilation line on the non-thermal spectrum. Thermal emission is much suppressed owing to plasma frequency effects of quark matter and the electrosphere [24]. Further cooling to temperatures $T<10^{8} \mathrm{~K}$ results in a non-thermal spectrum dominated by bremsstrahlung photons from electron-electron collisions in the electrosphere [14].

As mentioned in the introduction, a magnetic field is expected to exist at surface, altering the electrosphere profile and its emission properties. The electron motion perpendicular to the magnetic field is quantized into Landau orbitals enumerated by the quantum number $n_{L}=0,1,2 \ldots$. For a relativistic fermion of charge $q$ and mass $m$, such as the quarks and electrons in our case, the energy in a $\hat{z}$-directed magnetic field $B$ is given by

$$
\epsilon_{n}=\sqrt{m^{2} c^{4}+c^{2} p_{z}^{2}+2 m c^{2} \hbar \omega_{B} n} \equiv m c^{2} E ; \quad 2 n=2\left(n_{L}+\frac{1}{2}+\sigma\right) ; \quad \omega_{B}=\frac{q B}{m c},
$$

where $\sigma= \pm 1 / 2$ is the spin-degeneracy of the fermion. The $n=0$ state is non-degenerate while $n>0$ states are doubly degenerate. We express the magnetic field strength in convenient units ' $b$ ' such that

$$
b \equiv \frac{\omega_{B}}{m c^{2}}=\frac{B}{4.414 \times 10^{13} \mathrm{G}} \quad \text { for electrons }
$$

and take $B=4.414 \times 10^{13} \mathrm{G}$ (corresponding to $b=1$ ) as an upper limit on the surface magnetic field of quark stars. Although higher fields may exist in magnetars and AXPs (anomalous X-ray pulsars), their interior magnetic fields would likely be large enough to inhibit the formation of quark matter [25]. For a given magnetic field, both temperature and matter density determine the filling fraction of the Landau levels, and hence the degree of quantization. From Eqn.(3), the highest Landau level occupied by a fermion with energy $\epsilon_{n}$ is

$$
n_{L, \max }: n_{\max }=\operatorname{Int}(\nu) ; \quad \nu=\frac{E^{2}-1}{2 b} .
$$

Since the quarks are highly relativistic, $E \gg 1$ and many Landau levels are occupied for the light quarks, and even more for the strange quark, so that quantization effects may be ignored. Further, when the temperature $T \gg T_{B}$ where $T_{B}=\hbar \omega_{B} / k_{B}\left(k_{B}\right.$ is the Boltzmann constant), thermal effects smear out adjacent Landau levels, and the magnetic field is effectively non-quantizing, irrespective of the degeneracy. This effect is more important for quarks, which have a larger mass, and hence smaller cyclotron frequency, than electrons. We thus expect no modifications to the quark distribution due to magnetic fields of typical magnitudes considered in this work.

For electrons, quantization effects become important as we move outward from the electrosphere, and more electrons reside in the lower Landau levels. The number density of electrons in a magnetic field is given by

$$
n_{e}=\frac{m_{e} \omega_{B}}{h^{2}} \int_{-\infty}^{\infty} d p_{z} \sum_{n, \sigma} f\left(\epsilon_{n}\right) ; \quad f\left(\epsilon_{n}\right)=\frac{1}{1+\mathrm{e}^{\left(\epsilon_{n}-\mu_{e}\right) / T}}
$$

For a cold degenerate electron gas which satisfies $T \ll \mu_{e}$, we may approximate

$$
n_{e}\left(\mu_{e 0}\right)=\Theta\left(\mu_{e 0}-1\right) \frac{2 m_{e}^{2} \omega_{B} c}{h^{2}}\left[\sqrt{\mu_{e 0}^{2}-1}+2 \sum_{n=1}^{n_{\max }} \sqrt{\mu_{e 0}^{2}-1-2 b n}\right] ; \quad \mu_{e 0}=\frac{\mu_{e}(T=0)}{m_{e} c^{2}} .
$$


From Eqn.(7), the density at which the second Landau level becomes populated is

$$
n_{e}^{*}=\frac{\left(m_{e} \omega_{B}\right)^{3 / 2}}{\hbar^{2} \pi^{2} \sqrt{2}}=1.24 \times 10^{30} b^{3 / 2} \mathrm{~cm}^{-3} .
$$

This is already less than the typical electron density at surface in the $B=0$ case, where $n_{e} \sim$ $10^{-4}-10^{-3} / \mathrm{fm}^{3}[26]$. Therefore, several Landau levels will be occupied, and the $B$-field is non-quantizing. In fact, for $\nu \gg 1$ and $T \ll \mu_{e}$, it follows from Eqn.(7) that [27]

$$
n_{e}=\frac{2 m_{e}^{2} \omega_{B} c}{h^{2}} \sqrt{\mu_{e 0}^{2}-1}\left[\frac{2 \sqrt{\mu_{e 0}^{2}-1}}{3 b}-3\right] \approx \frac{\left(\mu_{e}^{2}-m_{e}^{2}\right)^{3 / 2}}{3 \pi^{2}},
$$

which is the usual expression relating the number density and chemical potential for a relativistic degenerate Fermi gas in the $B=0$ case. For the range of temperatures and densities considered in this work, $T \ll T_{B}=\hbar \omega_{B} / k_{B}$ holds, so that temperature effects on electron occupation numbers is negligible. The situation is summarized in Fig. 1, which shows how the number density of electrons, as obtained from Eqn.(6) behaves as a function of electron chemical potential, temperature and magnetic field. The truncation of the curves at $\nu=0$ for finite $T$ represent the fact that for lower (negative) chemical potentials, and hence densities, all electrons reside in the $n=0$ state. We note that only for temperatures $T \geq T_{B}$ does the number density deviate significantly from the $T=0$ curve (NB. This is due to the smearing of the Landau levels by temperature fluctuations.). We therefore continue to use the zero temperature approximation to the electron density in the rest of the paper.

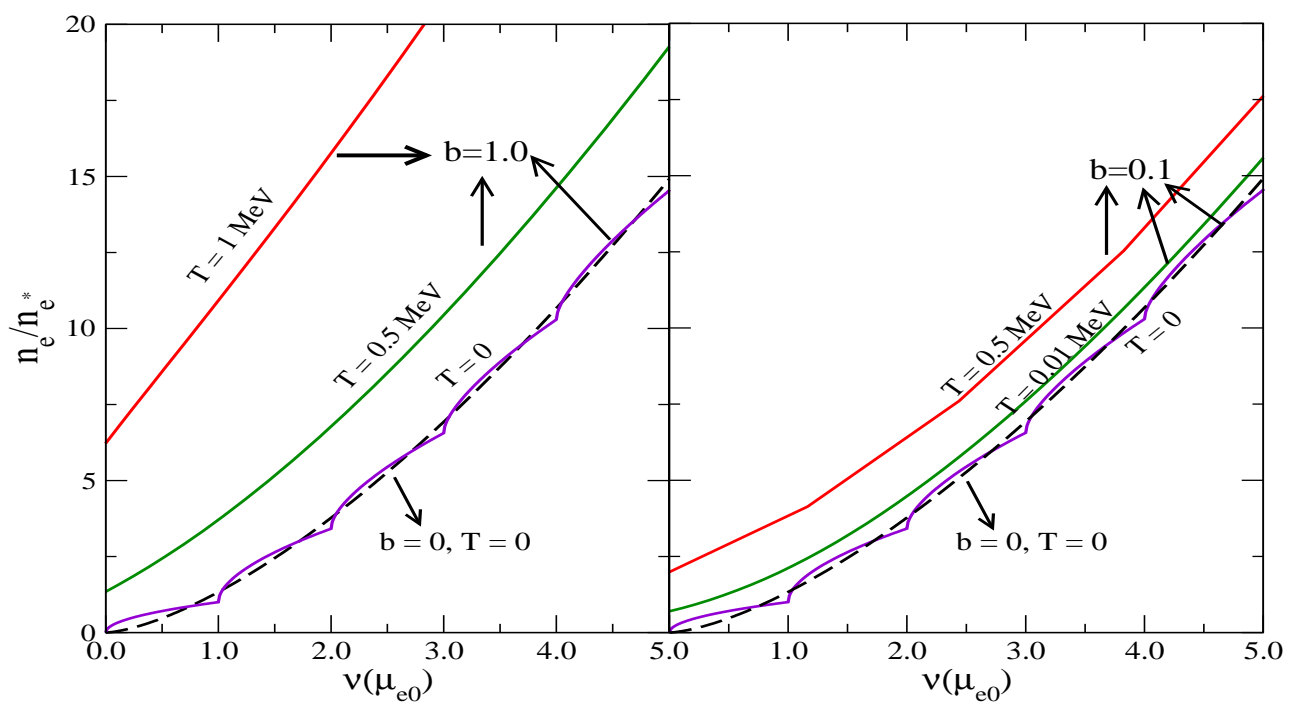

FIG. 1. The number density of electrons (scaled to $n_{e}^{*}$ ) from Eqn. (6) as a function of the filling factor $\nu$ for various temperatures. The solid curves are for non-zero magnetic field, and display a loss of the Landau level structure with increasing temperature.

\section{ELECTROSPHERE PROFILE WITH A MAGNETIC FIELD}

We would now like to obtain the electron density profile as a function of distance in the electrosphere. This will be modified from previous results $[11,24]$ which were in the absence of a magnetic field. The baryon density at the surface of a strange star, where the quark pressure vanishes, is expected to be in the range $(2-5) \rho_{0}$, corresponding to quark chemical potentials in the range $300-400 \mathrm{MeV}$. At such densities, the strange quark current mass is not ignorable, with $m_{s} \sim 150 \mathrm{MeV}$. Local charge neutrality then implies an electron chemical potential at surface $\mu_{e} \approx 15-20 \mathrm{MeV}$. This picture of a homogeneous surface as opposed to a crusty one with quark nuggets is justified by the large value of the surface tension given 
our parameter values [28]. The surface depletion of strange quarks at the surface results in a positively charged layer, which is two orders of magnitude thinner than the electrosphere. For cold electrospheres, this charged layer results in oppositely directed electric fields at the star's surface as well as an enhanced electron number density $n_{e} \gg n_{*}$.

For a general magnetic field with poloidal and toroidal components at the surface, the electron distribution can, in principle, be obtained using the formalism of magnetic field density functional theory or by Green function techniques, both of which are complicated tasks. As a first study, we adopt a simple picture of a constant magnetic field directed normally to the surface and ignore Coulomb interactions and exchange effects, which are justified approximations given the extreme degeneracy being explored in the problem. We employ the self-consistent Thomas-Fermi approximation and present a numerical solution of the Poisson equation

$$
\nabla^{2} \phi(\vec{r}, \vec{B})=-\rho(\vec{r}, \vec{B})=e n_{e}(\vec{r}, \vec{B}) .
$$

In the plane parallel approximation $\vec{B}=B \hat{z}, \hat{r}=\hat{z}$, and equilibrium requires $\mu_{e}\left(n_{e}(z)\right)-e \phi(z)=0$. For arbitrary magnetic fields, the equilibrium constraint involves magnetic forces $F_{\text {mag }}=\vec{j} \times \vec{B} \neq 0$ where $\vec{j}$ is the electron current density. With our approximations, the problem is an electrostatic one, and the electrostatic potential outside $(z>0)$ the star solves

$$
\begin{aligned}
\nabla^{2} \eta_{+} & =\frac{2 \alpha b m_{e}^{2}}{\pi} \Theta\left(\eta_{+}-1\right)\left[\sqrt{\eta_{+}^{2}-1}+2 \sum_{n=1}^{N} \sqrt{\eta_{+}^{2}-1-2 b n}\right] ; \quad N=\operatorname{Int}\left(\frac{\eta_{+}^{2}-1}{2 b}\right), \\
\eta_{+} & =\frac{e \phi_{+}}{m_{e} c^{2}} ; \quad \phi_{+}=\phi(z>0) .
\end{aligned}
$$

In the limit $N \gg 1$, when the electrons populate several levels, we recover the expression for the non-magnetic case

$$
\nabla^{2} \phi_{+}=\frac{4 \alpha}{3 \pi} e^{2} \phi_{+}^{3}
$$

while for $N=0$, we obtain

$$
\nabla^{2} \phi_{+}=\frac{e m \omega_{B} p_{F}}{2 \pi^{2}}
$$

the expression which has been derived previously for the non-relativistic case [29] but applies equally well to the relativistic case as long as only the $0^{\text {th }}$ level is populated. It is interesting to note that the above equation leads to an exponentially decaying electron profile at large distances from the surface, contrary to the $1 / z$ fall-off in the non-magnetic case. As $\eta_{+} \rightarrow 1$, we expect interactions and exchange effects to be important, but since this is only a minute region of the electrosphere, we do not correct for it.

Eq. (11) can be solved numerically once the boundary conditions are determined. Clearly, $\eta_{+} \rightarrow 1$ as $z \rightarrow \infty$ (this corresponds to vanishing electron number density and is numerically implemented by the shooting method to obtain the correct asymptote at sufficiently large $z$. This method is also practically applicable to the case of suspended crusts above a quark star; see Ref. [16].). The second boundary condition is determined at the surface $z=0$, where $\phi=\phi_{0} \Rightarrow \eta=\eta_{0}$ and $\eta_{0}$ must be determined by the discontinuity in the electric field $\nabla \phi_{+}-\nabla \phi_{-}=4 \pi \sigma$ with $\phi_{-}=\phi(z<0)$. The electrostatic potential $\phi_{-}$ inside the star is almost identical to the non-magnetic case since the electron density deep inside the star, given by $\tilde{\mu_{e}}=m_{s}^{2} / 4 \mu_{q} \sim 10 \mathrm{MeV} \gg m_{e} c^{2}=0.5 \mathrm{MeV}$ so that for $b \leq 10.0$ fields, quantization effects are negligible. In this case, $\phi_{-} \rightarrow \phi_{q}$ as $z \rightarrow-\infty$ where the relation between $\phi_{0}$ and $\phi_{q}$ is given by

$$
4 \pi \sigma=e\left(\frac{2 \alpha}{3 \pi}\right)\left[\phi_{0}^{2}+\left(\phi_{q}-\phi_{0}\right)\left\{\left(\phi_{0}+\phi_{q}\right)^{2}+2 \phi_{q}^{2}\right\}\right]^{1 / 2}
$$

wherein the prefactor corrects an error in equation (30) of Ref. [21]. For our choice of $\tilde{\mu}_{e}=11.25$ $\mathrm{MeV}$ (corresponding to $m_{s}=150 \mathrm{MeV}$ and $\mu_{q}=500 \mathrm{MeV}$ ), $\phi_{q}=37.4 \mathrm{MeV}, \phi_{0}=578 \mathrm{MeV}$ and hence $\eta_{0}=341 \mathrm{MeV}$. The boundary conditions having been specified, the results for the electrostatic potential $\phi_{+}, \phi_{-}$as obtained from Eq.(10) are illustrated in Fig. 2, and compared to the corresponding expressions for the non-magnetized electrosphere with the same boundary conditions. 

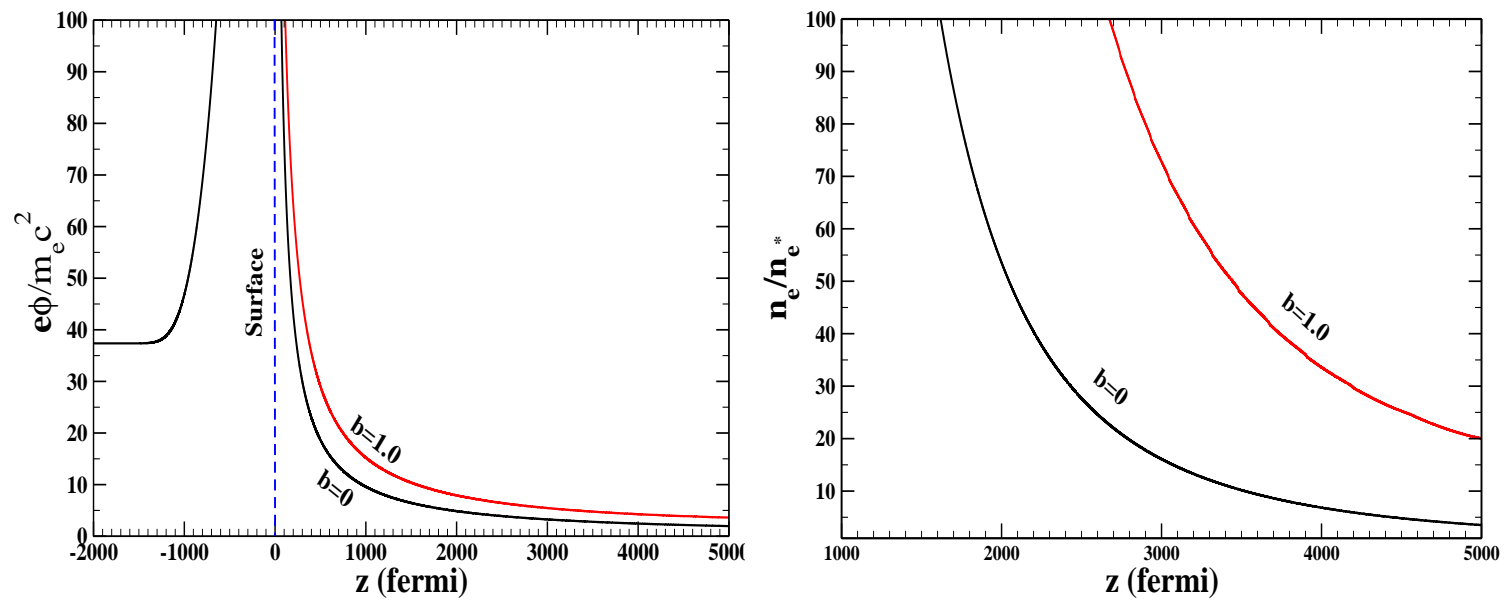

FIG. 2. Left panel: The electrostatic energy (scaled to $m_{e} c^{2}$ ) at the edge of the star, from Eqn. (10) for the unmagnetized $(b=0)$ and magnetized $(b=1.0)$ cases. Right panel: The corresponding electron number density (scaled to $n_{e}^{*}$ ) at the surface.

We observe that while the electrostatic potential inside the star is unaffected by magnetic field strengths of $b \sim \mathcal{O}(1)$, that outside the star is increased in comparison to the unmagnetized case. A similar finding for the number density reflects the fact that the electrons become localized in configuration space, their wave functions compressed into the Larmor radius, so that the Pauli repulsion is decreased and electrons can be squeezed together in greater numbers than in the unmagnetized case. This effect is most pronounced when the quantization effects are large, as can be seen by the wiggles that demarcate the filling of the Landau levels (right panel of Fig. 2). In both figures above, the curves meet at the surface $(z=0)$ where they satisfy the same boundary condition. At distances far from the surface, the curves cross each other since the total number density is the same in both cases. The number density falls off exponentially for the magnetized case while it falls off as $1 / z$ for the unmagnetized case.

In the absence of a thin $\left(10^{-5} \mathrm{M}_{\odot}\right)$ crustal layer of accreted matter above the strange star, the spectral properties of a strange star are linked to radiative transport in the electrosphere. This is because radiation from the stellar interior is cutoff by the high plasma frequency $\left(\omega_{p} \sim 20 \mathrm{MeV}\right)$ of dense quark matter, and Landau-Pomeranchuk-Migdal (LPM) suppression of bremsstrahlung photons from quarkquark collisions [24]. For an unmagnetized electrosphere, the emission at high luminosities is dominated by $e^{+} e^{-}$pair emission so long as the temperature is high enough. At lower luminosities, the emission is dominated by photon bremsstrahlung from electron-electron collisions in the electrosphere. The spectrum is non-thermal due to the finite plasma frequency of the degenerate electron gas and large mean free path for photons in the electrosphere.

The effect of the intense magnetic field on electrospheric emission is currently being investigated and will be reported elsewhere; here, we only state some qualitative effects. The plasma frequency plays a vital role in the emission properties of the electrosphere, since lower frequency photons are rapidly damped by the complexion of the refractive index

$$
n_{r}^{2}=1-\frac{\omega_{p}^{2}}{\omega^{2}} ; \quad \omega_{p}^{2} \simeq \frac{e^{2}}{3 \pi^{2}} \mu_{e}^{2}
$$

This collective effect of the plasma is important at high electron density (inner regions of the electrosphere) where the Debye length is smaller than the mean free path. Note that despite the large electron density, the large degeneracy limits the available scattering states of electrons, leading to a large mean free path for photons. In the presence of a magnetic field, when quantization effects become important, collective effects are determined by the Larmor radius rather than the Debye length, and the reflectivity for normal incidence becomes [30]

$$
R=\frac{1}{2}\left[\left|\frac{n_{r, 1}-1}{n_{r, 1}+1}\right|^{2}+\left|\frac{n_{r, 2}-1}{n_{r, 2}+1}\right|^{2}\right] ; \quad n_{r\{1,2\}}^{2}=1-\frac{\omega_{p}^{2}}{\omega^{2}} \frac{\omega}{\left(\omega \pm \omega_{B}\right)} .
$$


When $\omega_{p} \ll \omega \ll \omega_{B}$, the reflectivity is considerably diminished from the unmagnetized case, and the electron plasma will radiate more strongly. Further, the dielectric tensor will modify the Poisson equation, resulting in the breakdown of the plane parallel approximation employed here. Thus, our estimate of the profile is simplistic and must be made self-consistent. The intense magnetic field will also lead to polarization effects. Taking into account the thermal conductivity of the electrons in a strong magnetic field, a non-uniform surface temperature distribution is expected. The inclusion of toroidal components of the magnetic field can smoothen out some of these non-uniformities, and has been linked to the largely uniform blackbody spectrum of some isolated neutron stars [31]. It would be interesting to see if the electrospheric surface of the strange star displays similar spectral features. In particular, the possibility of having bound positronium in the magnetized electrosphere should be investigated.

\section{STRANGE STARS AND ASTROPHYSICS: THE QUARK-NOVA AND THE $R$-PROCESS}

Strange quark stars have been linked to some of the most energetic and violent phenomena in astrophysics, such as Gamma-ray bursts (GRBs) [32], type I X-ray superbursts [33] and Soft-gamma repeaters (SGRs) [34] in what is being perceived as a new paradigm in astrophysics - the connection of high-energy astrophysics with exotic forms of dense matter. The energy released in the phase conversion of a hadronic star to a quark star is typically about $10^{53} \mathrm{ergs}$, similar in magnitude to the energy of observed gammaray bursts, and accretion and flash heating on a strange star surface is a viable model for SGR flares and superbursts. Further, the emission spectra of the long bursts can also be explained by invoking the presence of strange quark matter and its subsequent transition to a color-superconducting state [35]. All of these scenarios are predicated upon the existence of absolutely stable three-flavor quark matter at high density.

Here, we discuss the formation of a quark star in a Quark-Nova, a scenario in which nuclear matter at high density in the core of a neutron star undergoes a phase transition to two flavor (up and down) quark matter, which then undergoes equilibrating weak reactions to form three flavor quark matter. The advantage is that this does not require the improbable simultaneous and spontaneous conversion to strange matter (via weak interactions) of several neutrons within a small volume. In addition, the Coulomb barrier-free absorption of neutrons can enlarge the quark phase, so that the whole neutron star is essentially converted to strange quark matter, thus forming a quark star. The dynamics of this process is only beginning to be explored [36], and it is likely that some mass ejection from the surface of a neutron star can take place, aided by neutrinos [37] but powered mostly by pre-compression shock waves from the rapidly advancing quark conversion front [38].

There are various ways in which this conversion can be triggered. A rapidly rotating neutron star is slowed down principally by magnetic braking (and additionally by energy loss through gravitational waves), thereby reducing the centrifugal forces and increasing central pressures. The timescales and dynamics of this spin-down have been discussed in [39]. The probability of triggering a first order transition by forming a tiny lump of two flavor quark matter (quantum nucleation) is exponentially sensitive to the central pressure [40]. This makes the transition much more likely as the star spins down. Material accumulated from a fallback disk around a cooling neutron star or accretion from a companion star, for example, in a low-mass X-ray binary system can also force the conversion. Even the Bondi accretion rate of $10^{21} \mathrm{~g} / \mathrm{sec}$ from the interstellar medium can lead to a mass increase of up to $0.1 M_{\odot}$ within a million years which can be sufficient to compress the star beyond the minimum density for the phase transition. Other possibilities include the clustering of $\Lambda$-baryons at high density and seeding by energetic cosmic neutrinos or strangelets [11]. Deconfinement of quarks can also occur as early as the protoneutron star stage following a core-collapse supernova explosion if the central density is large enough. However, in this case, neutrino trapping in the hot and dense interior can push the transition density higher, delaying the collapse to the point where a black hole might be formed instead [41].

The amount of mass ejected and the dynamics of the Quark-nova make it a potential candidate for $r$-process nucleosynthesis. The decompressing neutron matter at the surface of the neutron star which is undergoing the phase conversion to a quark star will expand rapidly and chunks of neutron matter will fall apart, allowing beta-decays to heat the material to close to $r$-process like temperatures of $T \sim 0.1$ $\mathrm{MeV}$. Following the expansion of this dense ejecta (including $\beta$-decays) down to neutron drip density using network calculations of Meyer [42], we find that the matter ends up distributed over a wide range of elements from $Z \sim 40-70$. At this stage, the full $r$-process reaction network can be coupled to 
obtain the final abundance distribution, as well as the evolution of temperature, entropy and electron fraction. The most important parameter that governs the evolution of the above physical quantities is the expansion timescale of the ejecta, which is the time taken to evolve from a density of $\rho \simeq 10^{14} \mathrm{~g} / \mathrm{cc}$ to $\rho \simeq 10^{11} \mathrm{~g} / \mathrm{cc}$. Assuming that the ejecta is on an escape trajectory and expands as a multiple of the free-fall timescale in a spherically symmetric, non-interacting manner, we obtain a timescale on the order of 0.01-1 milliseconds. Fig. 3 shows the $r$-abundance of the elements (labeled "network") from a QuarkNova compared to the normalized solar $r$-abundance pattern. The overabundance for elements with mass number $A>230$ is due to the omission of alpha-decays in the network. It is also notable that not all neutrons are consumed in this scenario, and that several light elements are produced with significant abundance. It is likely that Quark-Novae may be an additional contributor to the $r$-process material in the universe, presenting another link between dense quark matter and astrophysics. A detailed analysis of $r$-process nucleosynthesis from Quark-Novae, including contrasts to standard or primary astrophysical sites such as Type II supernovae and neutron star mergers, and comparisons to trends of heavy-element abundance ratios in metal-poor stars will be presented in a forthcoming article [38].

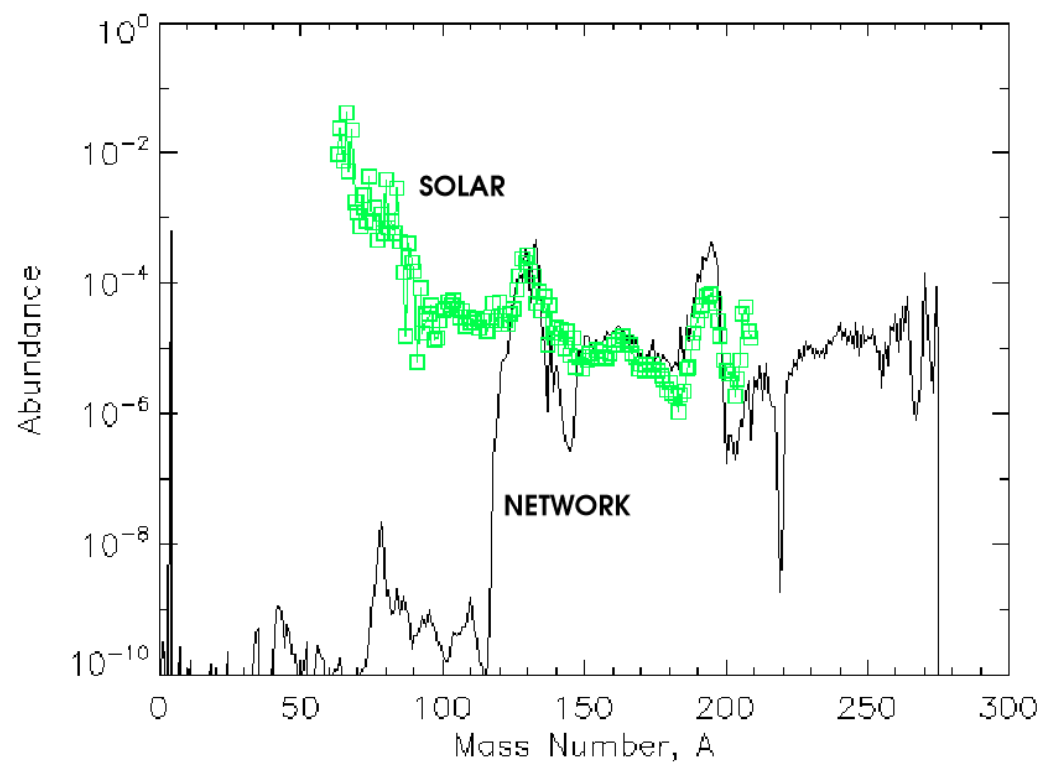

FIG. 3. Abundance pattern of $r$-process elements from a Quark-Nova: The initial electron fraction $Y_{e}=0.03$, entropy per nucleon $S / k_{B}=1$, temperature $T_{9}=1.0$ and expansion timescale $\tau=1.0$ millisecond. The scaled comparison to solar abundance is also shown.

While several observable characteristics of strange stars have been conjectured in the literature [43,44], such as period-clustering, anomalies in braking index, global properties (mass and radius), and small spin-periods, spectral differences between quark stars and neutron stars have only been touched upon. If strange stars are made in Quark-Novae, and are linked to $r$-processing of the heavy elements, one can expect a most direct evidence of a quark star to come from $\gamma$-decays of unstable nuclei produced in the $r$-process. We expect the Quark-Nova ejecta to achieve $\gamma$-ray transparency sooner than supernova ejecta since Quark star progenitors (i.e., neutron stars) lack extended atmospheres, so that $r$-process only gamma emitters with lifetimes of the order of years (such as ${ }^{137} \mathrm{Cs},{ }^{144} \mathrm{Ce},{ }^{155} \mathrm{Eu}$ and ${ }^{194} \mathrm{Os}$ ) can be used as tags for the Quark-Nova.

In conclusion, this is an exciting time for quark star research. Recent developments in the theory of high density quark matter have provided novel ways of explaining the most energetic astrophysical events, and spurred re-examinations of old paradigms of strange stars. With increasing data now coming from myriad sources (egs. neutron stars that push theoretical limits on spin periods and radius; GRB spectra that link to quark stars as inner engines; abundance trends of heavy elements in ultra metal-poor stars that point to multiple sources of the $r$-process), it appears that quarks may play an important role in uncovering the secrets of many astrophysical puzzles. 


\section{ACKNOWLEDGMENTS}

I thank the organizers of the IXth workshop on high-energy physics and phenomenology (WHEPP-9) at the Institute of Physics, Bhubaneswar, India, for hosting an exciting meeting in a most hospitable setting. I acknowledge useful interactions with Kaya Mori, Rachid Ouyed, Kaori Otsuki, Brad Meyer, Mandar Bhagwat and Stewart Wright, and the hospitality of the Canadian Institute for Theoretical Astrophysics (CITA), where discussions pertinent to this work were held. P.J. is supported by the Department of Energy, Office of Nuclear Physics, contract no. W-31-109-ENG-38.

[1] J. Schaffner-Bielich, J. Phys. G31, S561 (2005).

[2] A. R. Bodmer, Phys. Rev. D4, 1601 (1971); H. Terazawa, INS-Report-338, University of Tokyo (1979); E. Witten, Phys. Rev. D30, 272 (1984).

[3] R. Xu, in Proceedings of The 2005 Lake Hanas International Pulsar Symposium, astro-ph/0512519.

[4] S. Chin and A. K. Kerman, Phys. Rev. Lett 43, 1292 (1979).

[5] M. Alford, M. Braby, M. Paris and S. Reddy, Astrophys. J. 629, 969 (2005).

[6] M. Alford, J. A. Bowers and K. Rajagopal, J. Phys. G27, 541 (2001).

[7] I. A. Shovkovy, M. Hanauske and M. Huang, hep-ph/0310286.

[8] E. J. Ferrer, V. de la Incera and C. Manuel, hep-ph/0601179.

[9] D. M. Sedrakian and D. Blaschke, Astrofiz. 45, 203 (2002).

[10] A. Drago, A. Lavagno and G. Pagliara, Phys. Rev. D69, 057505 (2005).

[11] C. Alcock, E. Farhi and A. V. Olinto, Astrophys. J. 310, 261 (1986).

[12] A. G. Aksenov, M. Milgrom and V. V. Usov, Astrophys. J. 609, 363 (2004).

[13] D. Page and V. V. Usov, Phys. Rev. Lett. 89, 131101 (2002).

[14] P. Jaikumar, C. Gale, D. Page and M. Prakash, Phys. Rev. D70, 023004 (2004).

[15] J. Knodlseder, astro-ph/0207527.

[16] M. Stejner and J. Madsen, Phys. Rev. D72, 123005 (2005).

[17] P. Jaikumar, S. Reddy and A. Steiner, Phys. Rev. Lett. 96, 041101 (2006).

[18] M. Alford, K. Rajagopal, S. Reddy and A. Steiner, hep-ph/0604134.

[19] R. Ouyed, B. Niebergal, W. Dobbler and D. Leahy, astro-ph/0510691.

[20] J. F. Pérez-Azorín, J. A. Miralles and J. A. Pons, astro-ph/0510684.

[21] V. V. Usov, K. S. Cheng and T. Harko, Astrophys. J. 620, 915 (2005).

[22] J. Madsen, Phys. Rev. Lett. 87, 172003 (2001).

[23] J. Schwinger, Phys. Rev. 82, 664 (1951).

[24] K. S. Cheng and T. S. Harko, Astrophys. J. 622, 1033 (2005).

[25] S. Ghosh and S. Chakrabarty, Pramana 60, 901 (2002).

[26] V. V. Usov, Astrophys.J. 550, L179 (2001).

[27] A. M. Abrahams and S. L. Shapiro, Astrophys. J. 374, 652 (1991).

[28] M. S. Berger and R. L. Jaffe, Phys. Rev. C35, 213 (1987).

[29] I. Fushiki, E. H. Gudmundsson and C. J. Pethick, Astrophys. J. 342, 958 (1989).

[30] M. van Adelsberg, D. Lai, A. Y. Potekhin and P. Arras, Astrophys. J. 628, 902 (2005).

[31] U. Geppert, M. Küker and D. Page, Astron. \& Astrophys. 426, 267G (2004); see also astro-ph/0512530.

[32] B. Paczynski and P. Haensel, MNRAS 362, L4 (2005).

[33] D. Page and A. Cumming, Astrophys.J. 635, L157 (2005).

[34] V. V. Usov, Phys. Rev. Lett. 87, 021101 (2001).

[35] R. Ouyed, R. Rapp and C.Vogt, Astrophys. J. 632, 1001 (2005).

[36] A. Drago, A. Lavagno and I. Parenti; astro-ph/0512652

[37] P. Jaikumar, R. Ouyed and P. Keränen, Astrophys. J. 618, 485 (2004).

[38] P. Jaikumar, R. Ouyed, K. Otsuki and B. S. Meyer, in preparation.

[39] J. E. Staff, R. Ouyed and P. Jaikumar, astro-ph/0603743.

[40] I. Bombaci, I. Parenti, and I. Vidana, Astrophys. J 614, 314 (2004).

[41] M. Prakash, I. Bombaci, M. Prakash, P. J. Ellis, J. M. Lattimer and R. Knorren, Phys. Rep. 280, 1 (1997).

[42] B. S. Meyer, Astrophys. J. 343, 254 (1989).

[43] N. K. Glendenning, Compact Stars, $2^{\text {nd }}$ ed. (Springer-Verlag, New York).

[44] F. Weber, Prog. Part. Nucl. Phys. 54, 193 (2005). 